ITC 2/48

Journal of Information Technology and Control

Vol. 48/ No. 2/ 2019

pp. 357-365

DOI 10.5755/j01.itc.48.2.21864
Recovery of a Compressed Sensing CT Image Using a Smooth

Re-weighted Function- Regularized Least-Squares Algorithm

\title{
Recovery of a Compressed Sensing CT Image Using a Smooth Re-weighted Function- Regularized Least-Squares Algorithm
}

\section{Peng-bo Zhou}

School of Art \& Communication, Beijing Normal University, Beijing 100875, China, e-mail: zhoupengbo@bnu.edu.cn

\section{Kang LI}

School of Computer Science and Technology, Norwest University, Xi'an 710048, China, e-mail: 854092@qq.com

\section{Wei Wei}

School of Computer Science and Engineering, Xi'an University of Technology, Xi'an 710048, China, e-mail:weiwei@xaut.edu.cn

\section{Marcin Woźniak}

Institute of Mathematics, Silesian University of Technology, Kaszubska 23, 44-100 Gliwice, Poland, e-mail:Marcin.Wozniak@polsl.pl

\section{Zhuo-ming Du}

School of Mathematical Sciences, Nanjing Normal University, Nanjing 210023, China, e-mail: duzhuoming423dzm@126.com

\section{Hong-an Li}

College of Computer Science and Technology, Xi'an University of Science and Technology, Xi'an 710054, China, e-mail:an6860@126.com 
It is challenging to recover the required compressed CT (Computed Tomography, CT) image, which is got by transferred through the internet or is stored in a signal library after being compressed. We present a recovery method for compressed sensing CT images. At present, minimizing 0-norm, 1-norm and p-norm is used to recover compressed sensing signals. However, sometimes 0-norm is an NP problem, 1-norm has no solution in theory and p-norm is not a convex function. We introduce a recovery method of compressed sensing signal based on regularized smooth convex optimization. In order to avoid solving the non-convex optimization problems and no solution condition, a convex function is designed as the objective function of optimization to fit 0-norm of signal and a fast iterative shrinkage-thresholding algorithm is proposed to find solution with the convergence speed is quadratic convergence. Experimental results show that our method has a sound recovery effect and is well suitable for processing big data of compressed CT images.

KEYWORDS: Compressed sensing; CT Image; Re-weighted function; Regularized Least-Squares; Sparse representation.

\section{Introduction}

CT (Computed Tomography, CT) images cannot only show physicians the anatomical structure of bones inside human body, but also reveal the damaged level to a certain degree. In hospital, physicians usually transfer CT images through the Internet or the local area network, but the big data of CT images pose a limit for the possibility of remote transmission or preview. Then these CT images are compressed before transmission or stored in a library and the CS (Compressed Sensing, CS) method is a popular compression method at present. These compressed images must be recovered if we want to make good use of them. So the compressed sensing $\mathrm{CT}$ image recovery becomes a hot research topic thanks to the rapid internet development. In this paper, we study how to recover the $\mathrm{CT}$ images which are compressed using the compressed sensing method. We could treat a sound signal as a 1-D signal, an image as a 2-D signal, and a 3-D model as a 3-D signal, so in this paper we treat CT images as 2-D signals. As an alternative and effective data acquisition strategy, the compressive sensing acquires a signal by collecting a relatively small number of linear measurements. Algorithms for signal recovery in a CS framework are referred to as sparse signal recovery (SSR) algorithms. Generally, the existing methods can be categorized as the 0-norm minimization, 1-norm minimization and $\mathrm{p}$-norm $(0<p<1)$ minimization. Unfortunately, 0-norm minimization is a combinatorial optimization problem whose computational complexity grows exponentially with the signal size. Recently, many approaches were developed based on minimizing 0-norm because it is the direct approach [1$2,6,10-14,19,22]$. 1-norm minimization is the most popular algorithms, which has been widely applied [22]. However, sometimes in theory, there will be an infinite number of solutions. p-norm $(0<p<1)$ minimization will solve the effect of 1-norm minimization. Several SSR algorithms based on constrained p-norm minimization with $(0<p<1)$ have also been proposed [1-2, 6-8, 10-14, 19, 22, 24-25].

In this paper, we propose a new algorithm for the recovery of compressed sensing CT images (2-D signals) and it is also suitable to the ordinary CS images. Furthermore, a new objective function is designed to replace signal's 0-norm, and a fast iterative shrinkage-thresholding algorithm is put forward to find the solution with a quadratic convergence speed.

\section{Preliminaries}

Recover compressed sensing signal is a linear inverse problem. It is a discrete linear system as:

$$
y=\Psi x+w,
$$

where, $\Psi \in R^{m \times n}(m<<n)$ and $y \in R^{m}, w \in R^{m}$ is an unknown noise vector. This linear system has a wide range of application prospect in many fields such as astrophysics, statistical inference, image processing 
or optics. If $x$ is sparse, the solution of $y=\Psi x+w$ can be obtained by solving the constrained optimization problem:

$$
x=\underset{x}{\operatorname{minimize}}\|y-\Psi x\|_{2}^{2}+\lambda\|x\|_{0} .
$$

Unfortunately, the optimizing $\|x\|_{0}$ is a combinatorial optimization problem whose computational complexity grows exponentially with the signal size $n$.

Recently, the widely used recovery method is the optimizing signal's 1-norm:

$$
x=\underset{x}{\operatorname{minimize}}\|y-\Psi x\|_{2}^{2}+\lambda\|x\|_{1} .
$$

The solving process of the optimizing signal's 1-norm is shown in Figure 1.

The $\mathrm{p}$-norm $(0<p<1)$ minimization recover a sparse signal from noisy measurements by solving the optimization problem:

$$
x=\underset{x}{\operatorname{minimize}}\|y-\Psi x\|_{2}^{2}+\lambda\|x\|_{p}^{p} .
$$

\section{Figure 1}

The solving process of optimizing signal's 1-norm

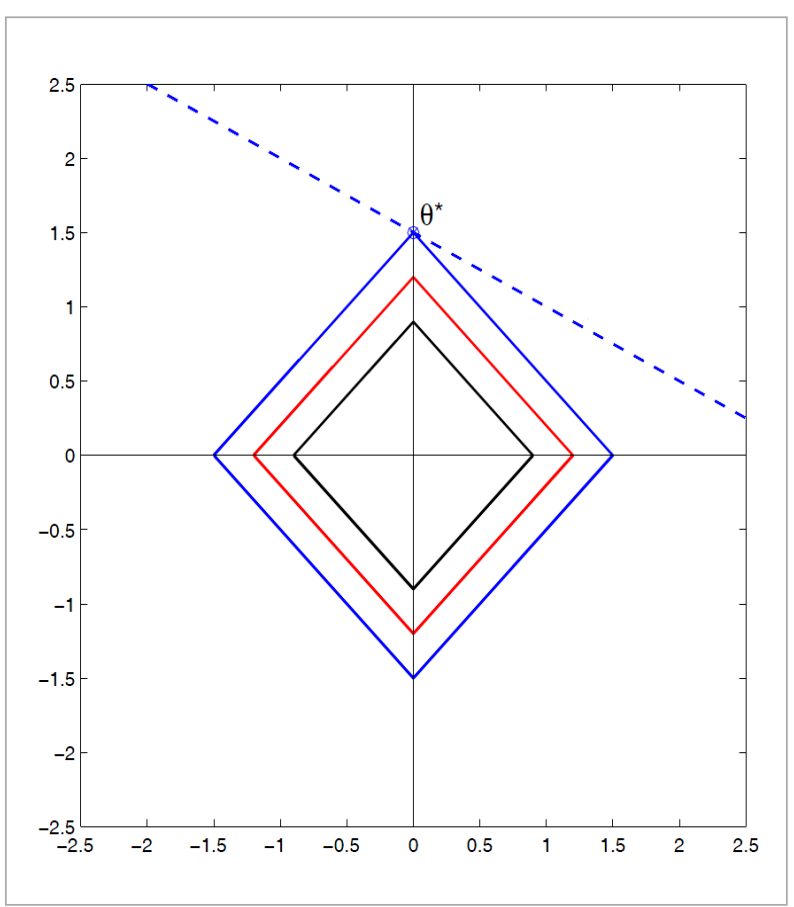

\section{Minimization of a Smooth Re-weighted Function-Regularized 2-Norm Error}

\subsection{Construction of Objective Function Fitting Signal's 0-Norm in the Whole Space}

It is known that the compressed sensing signal could be recovered directly by the minimizing 0 -norm. However, it falls into the class of NP problems. In this section, a new function $f$ fitting signal's 0-norm is presented and the signal recovery process is turned into the optimization function:

$$
f(x)=\sum_{i=1}^{n}\left[\frac{2}{\pi} \arctan \left(-e^{\beta^{*} x_{i}^{2}}+1\right)\right]^{2} .
$$

Then, based on this function, the result is obtained:

$-e^{\beta^{*} x_{i}^{2}}=-1$ when $x_{i}=0$ and then $-e^{\beta^{*} x_{i}^{2}}+1=0$;

$-e^{\beta^{*} x_{i}^{2}} \rightarrow-\infty$ when $x_{i} \neq 0$ and then $-e^{\beta^{*} x_{i}^{2}}+1 \rightarrow-\infty$.

$-e^{\beta^{*} x_{i}^{2}}+1$ is a monotonous decrease function, where $\beta$ is a fixed parameter and it is determined by specific issues.

From Figure 2, it is can be seen that $\arctan (\cdot)$ compresses the whole space to $\left(-\frac{\pi}{2}, \frac{\pi}{2}\right)$. $\arctan (\cdot)$ is convex in $(-\infty, 0)$ while it is concave in $(0, \infty) . \quad \frac{2}{\pi} \arctan \left(-e^{\beta^{*} x_{i}^{2}}+1\right)$ is a convex function for ${ }^{\pi}\left(-e^{\beta^{*} x_{i}^{2}}+1\right) \in(-\infty, 0)$ and $\frac{2}{\pi} \arctan \left(-e^{\beta^{*} x_{i}^{2}}+1\right) \in(-1,0)$.

Because $\frac{2}{\pi} \arctan \left(-e^{\beta^{*} x_{i}^{2}}+1\right) \in(-1,0)$, $\left[\frac{2}{\pi} \arctan \left(-e^{\beta^{*} x_{i}{ }^{2}}+1\right)\right]^{2} \in[0,1)$, it fits $\left|x_{i}\right|^{0}$.

Let $-z^{2}=\frac{2}{\pi} \arctan \left(-e^{\beta^{*} x_{i}^{2}}+1\right)$, then $\left[\frac{2}{\pi} \arctan \left(-e^{\beta^{*} x_{i}^{2}}+1\right)\right]^{2}=\left(-z^{2}\right)^{2}=z^{4}$. We can get $\frac{\mathrm{d}^{2}\left\{\left[\frac{2}{\pi} \arctan \left(-e^{\beta^{*} x_{i}^{2}}+1\right)\right]^{2}\right\}}{\mathrm{d} x_{i}^{2}}=12 z^{2} \geq 0$. 
Figure 2

The $\frac{2}{\pi} \arctan \left(-e^{\beta^{*} x_{i}^{2}}+1\right)$ image

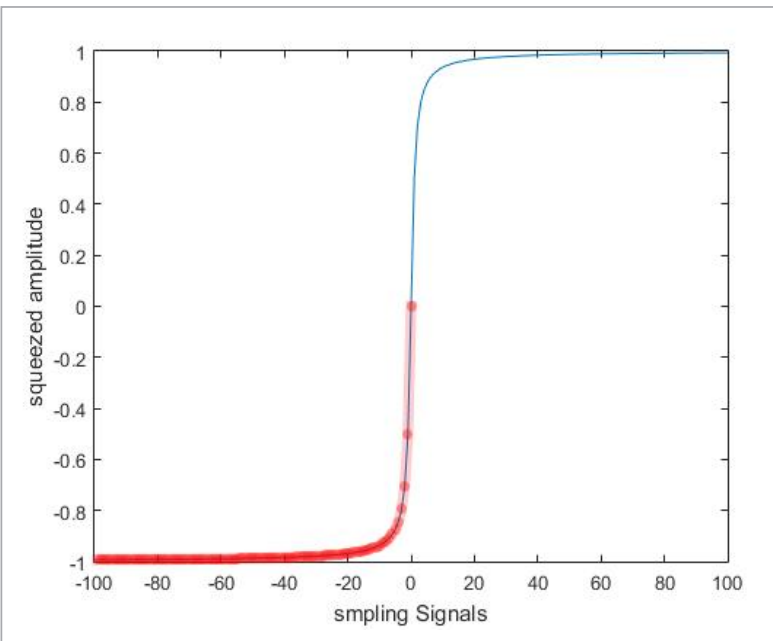

Thus, $\left[\frac{2}{\pi} \arctan \left(-e^{\beta^{*} x_{i}^{2}}+1\right)\right]^{2}$ is a convex function.

Because the linear combination of the convex function is still a convex function, so the function $f(\mathbf{x})=\sum_{i=1}^{n}\left[\frac{2}{\pi} \arctan \left(-e^{\beta^{*} x_{i}^{2}}+1\right)\right]^{2}$ is convex. At the same time, it fits $\|\mathbf{x}\|_{0}=\sum_{i=1}^{n}\left|x_{i}\right|^{0}$.

\subsection{Solution of the Unconstrained Optimization Problem}

We proposed to recover a sparse signal from noisy measurements by solving the optimization problem

$$
x=\underset{x}{\operatorname{minimize}}\left\{\begin{array}{l}
\sum_{i=1}^{n} \omega_{i}\left(y_{i}-x_{i}\right)^{2} \\
+\lambda \sum_{i=1}^{n} \omega_{i}\left[\frac{2}{\pi} \arctan \left(-e^{\beta^{*} x_{i}^{2}}+1\right)\right]^{2}
\end{array}\right\},(\text { (7) }
$$

where, $\omega_{i}=\frac{1}{x_{i}}$. The optimal solution for ( 7 ) is $x^{*}$. The solving $x^{*}$ process is: to solve a series of $\left\{x^{1} \quad x^{2} \quad \mathrm{~L} \quad x^{n}\right\}$, until $\left\|x^{n}-x^{*}\right\| \leq \varepsilon$.

According to the steepest descent method in the optimization theory, ( 7 ) becomes:

$$
x^{k}=\arg \min \left\{\begin{array}{l}
\frac{1}{2 t_{k}} \sum_{i=1}^{n} \omega_{i}\left(x_{i}-c_{k}^{i}\right)^{2} \\
+\lambda \sum_{i=1}^{n} \omega_{i}\left[\frac{2}{\pi} \arctan \left(-e^{\beta^{*} x_{i}^{2}}+1\right)\right]^{2}
\end{array}\right\},
$$

where, $c_{k}=x^{k-1}-t_{k} \nabla f\left(x^{k-1}\right)$.

Using the method of separation of variables, we transform the $\mathrm{n}$-dimensional primal problem to $\mathrm{n}$ one-dimensional problems.

$$
\begin{aligned}
x^{k}= & \min \omega_{1}\left\{\frac{1}{2 t_{k}}\left\|x_{1}-c_{k}\right\|_{2}^{2}+\lambda\left[\arctan \left(-e^{\beta * x_{1}{ }^{2}}+1\right)\right]^{2}\right\} \\
& +\min \omega_{2}\left\{\frac{1}{2 t_{k}}\left\|x_{2}-c_{k}^{2}\right\|_{2}^{2}+\lambda\left[\arctan \left(-e^{\beta * x_{2}{ }^{2}}+1\right)\right]^{2}\right\}+\ldots \\
& +\min \omega_{n-1}\left\{\frac{1}{2 t_{k}}\left\|x_{n-1}-c_{k}^{n-1}\right\|_{2}^{2}+\lambda\left[\arctan \left(-e^{\beta * x_{n-1}}+1\right)\right]^{2}\right\} \\
& +\min \omega_{n}\left\{\frac{1}{2 t_{k}}\left\|x_{n}-c_{k}^{n}\right\|_{2}^{2}+\lambda\left[\arctan \left(-e^{\beta * x_{n}{ }^{2}}+1\right)\right]^{2}\right\} .
\end{aligned}
$$

This problem can be used in the algorithm 1 . The algorithm enjoys a convergence rate of $O\left(K^{-2}\right)$ : $F\left(x^{k}\right)-F\left(x^{*}\right) \leq \frac{2 C(\nabla f(x))\left\|x^{0}-x^{*}\right\|_{2}^{2}}{(k+1)^{2}}[25]$.

Algorithm 1. The Fast Iterative Shrinkage-Thresholding Algorithm

Step 1. Let the starting point $x^{0}=0, t_{k}=\frac{1}{C(\nabla f(x))}$.

While, $\left(\left\|f\left(x_{k+1}\right)+g\left(x_{k+1}\right)-f\left(x_{k}\right)-g\left(x_{k}\right)\right\|\right) \leq \varepsilon$.

Step 2. $x_{k}=\Gamma_{\lambda t_{k}}\left(c_{k}\right)$

$\Gamma_{\lambda t_{k}}=\left(\left|c_{k}\right|-\lambda t_{k}\right)_{+} \operatorname{sgn}\left(c_{k}\right)$.

Step 3. $t_{k+1}=\frac{1+\sqrt{1+4 t_{k}^{2}}}{2}$.

Step 4. $x_{k+1}=x_{k}+\frac{t_{k}-1}{t_{k+1}}\left(x_{k}-x_{k-1}\right)$.

Step 5. $x^{k+1}$ is the solution.

\subsection{The Recovery Algorithm}

Algorithm 2. The recovery method of a compressed sensing signal 
Step 1. Recover a sparse signal by solving the optimization problem:

$$
x=\underset{x}{\operatorname{minimize}}\left\{\begin{array}{l}
\sum_{i=1}^{n} \omega_{i}\left(y_{i}-x_{i}\right)^{2} \\
+\lambda \sum_{i=1}^{n} \omega_{i}\left[\frac{2}{\pi} \arctan \left(-e^{\left.\beta^{*} x_{i}^{2}+1\right)}\right]^{2}\right.
\end{array}\right\}
$$

Step 2. Input $y, \Phi, D, \lambda, \beta, x_{0}=0$.

Step 3. Transform the optimal problem (10) into (7), and ( 7$)$ is solved by the Algorithm 1.

\section{Experimental Comparison and Analysis}

At first, 15 sparse signals $X$ of 256 dimensions with sparsity $(K=5 a-4) \quad a=1,2 \mathrm{~K}, 15$ are designed for the simulation experiment. The design method is described as follows: the first generate signal $u$ of the length $K$ according to the standard normal distribution; then select randomly $K$ as the indices of $X$ and insert $u$ into $K$ indices, and then sample $X$ by $\Psi_{m \times n}$ ( $m=100, n=256$ ) to get the compressed signal $y$. Finally, based on $y, X$ is recovered using Du's method [10], Pant's method [22], Donoho's method [22] and our method, respectively. Figure 3 shows the number

\section{Figure 3}

The number of successful recovery

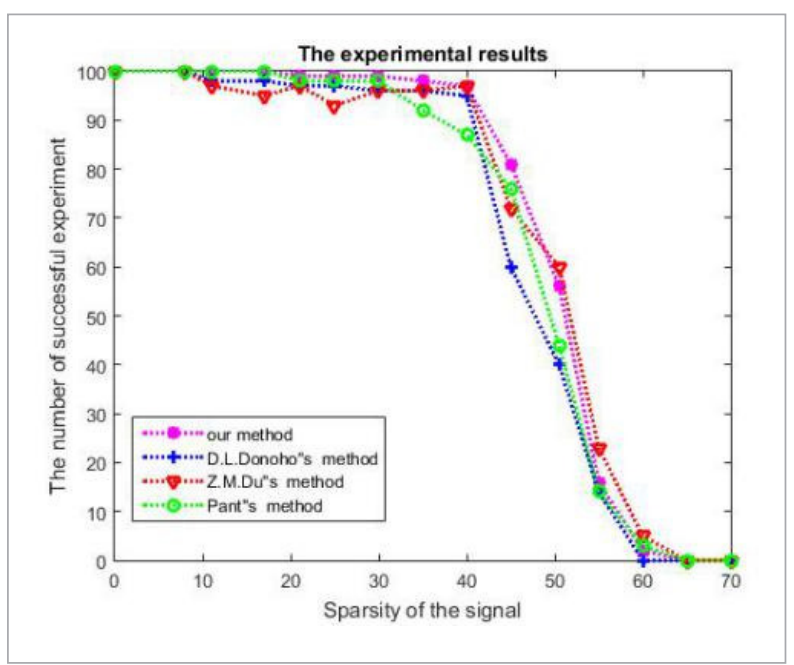

of successful recovery and Figure 4 shows the time required in the recovery [19-20, 22-25, 27, 31].

From Figure 3, we could observe when the sparsity is below 40 the numbers of successful recovery of the four methods are high, and our method is higher than the three methods. When the sparsity is above 40 the successful rates of four methods drop rapidly. Our method is a little higher and more stable than the other three methods [5, 9, 16, 18, 21, 26, 28, 32].

From Figure 4, on the aspect of the time required in the recovery our method is superior to the other three methods and the time growth of our method is nearly linear.

\section{Figure 4}

The CPU time required in the recovery

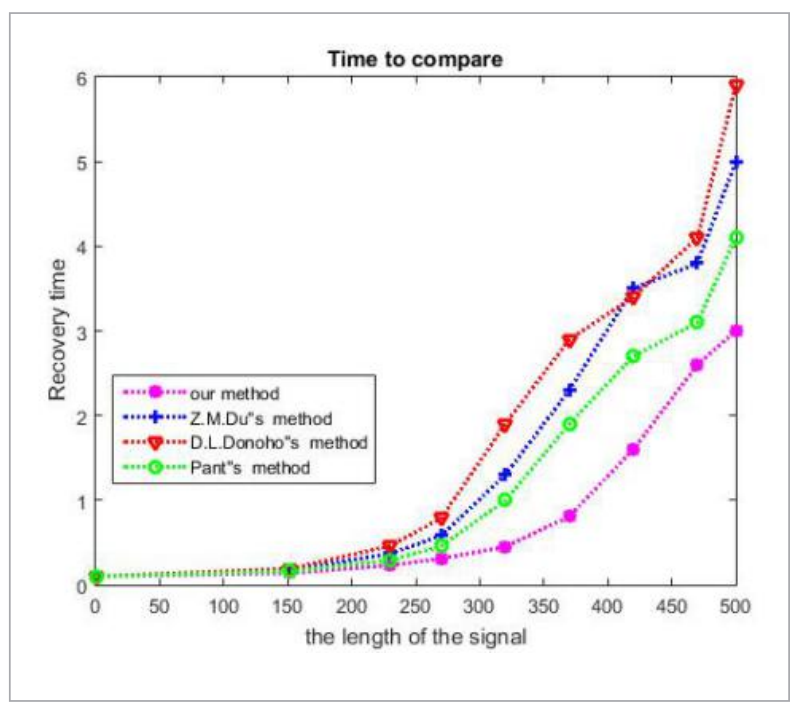

Secondly, a 2-D image is recovered in the same way. A 2-D image can be depicted as a sequence of 2-D points, and an original CT image is shown in Figure 5.

It can be represented as $\left[\begin{array}{ll}X & Y\end{array}\right]$, where, $X$ and $Y$ are vectors. Although most elements of $\left[\begin{array}{ll}X & Y\end{array}\right]$ are not zeros, which is sparse under the basis composed with eigenvectors of

\section{Figure 5}

A 2-D image is represented as a sequence of $2-D$ points

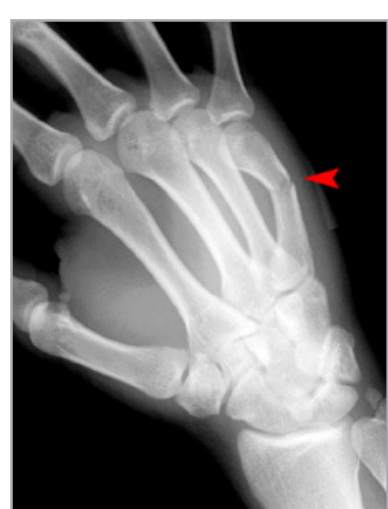


Laplace operator. The discrete Laplace operator is shown as follows:

$$
\delta(X)=L X=\left[\begin{array}{ccccccc}
1 & -\frac{1}{2} & 0 & \mathrm{~K} & \mathrm{~K} & 0 & -\frac{1}{2} \\
-\frac{1}{2} & 1 & -\frac{1}{2} & 0 & \mathrm{~K} & \mathrm{~K} & 0 \\
\mathrm{M} & \mathrm{M} & \mathrm{M} & \mathrm{M} & \mathrm{M} & \mathrm{M} & \mathrm{M} \\
0 & \mathrm{~K} & \mathrm{~K} & 0 & -\frac{1}{2} & 1 & -\frac{1}{2} \\
-\frac{1}{2} & 0 & \mathrm{~K} & \mathrm{~K} & 0 & -\frac{1}{2} & 1
\end{array}\right] X
$$

Figure 6 (the right-hand side) shows a histogram for the $\mathrm{x}$-coordinates of the CT image under the new basis and it is the spectral transform of 2-D image. As a result, 2-D image can be compressed based on compressed sensing.

Definition 1: Let $\mathbf{X}_{n \times n}=\left\{x_{i j} \mid i=1 \mathrm{~K} n, j=1 \mathrm{~K} m\right\}$, the Frobenius norm of $\mathbf{X}_{m \times n}$ is: $\|\mathbf{X}\|_{F}=\sqrt{\sum_{j=1}^{m} \sum_{i=1}^{n} x_{i j}{ }^{2}}$. Let the original CT image be $\mathbf{O}_{401 \times 2}$, the recovered model be $\mathbf{R}_{401 \times 2}$. Their similarity can be expressed as: $d=\left\|\mathbf{O}_{401 \times 2}-\mathbf{R}_{401 \times 2}\right\|_{F}$.
The CT image is sampled by $S_{76 \times 401}$ and $S_{50 \times 401}$, respectively. The applications of our method, pant's method, Z.M.Du method and Donoho's method to recovery of the image, are shown in Figure \%. We can see form Figure 7 , when the CT image is sampled by $S_{76 \times 401}$ the four methods all can recover the model perfectly. When the CT image is sampled by $S_{50 \times 401}$ the result of our method is superior to other three methods and the bone damage is even more clear than the original CT image. Donoho's method almost cannot recovery the model $(d=4.2)$, because $S_{50 \times 401}$ is not satisfied with the RIP (Restricted Isometric Property, RIP) condition. The objective function of our method is a convex function and a fast iterative shrinkage-thresholding algorithm is proposed to find the solution with a quadratic convergence speed. The robustness of our method is guaranteed by the objective function $[3,15,17-18$, 28-30, 33-34].

In fact, the data of CT image is big after being sampled using the traditional Shannon-Nyquist sampling method and the number of dimensions is twice the original signal. Efficiencies of many traditional compression methods are low because the data is compressed after being sampled. While, the compressed sensing method compresses the data in the sampling process. So, the shortcoming of the large volume of data is essentially overcome by the com-

\section{Figure 6}

$\mathrm{X}$-coordinates of the CT image and its new expression under the new bases
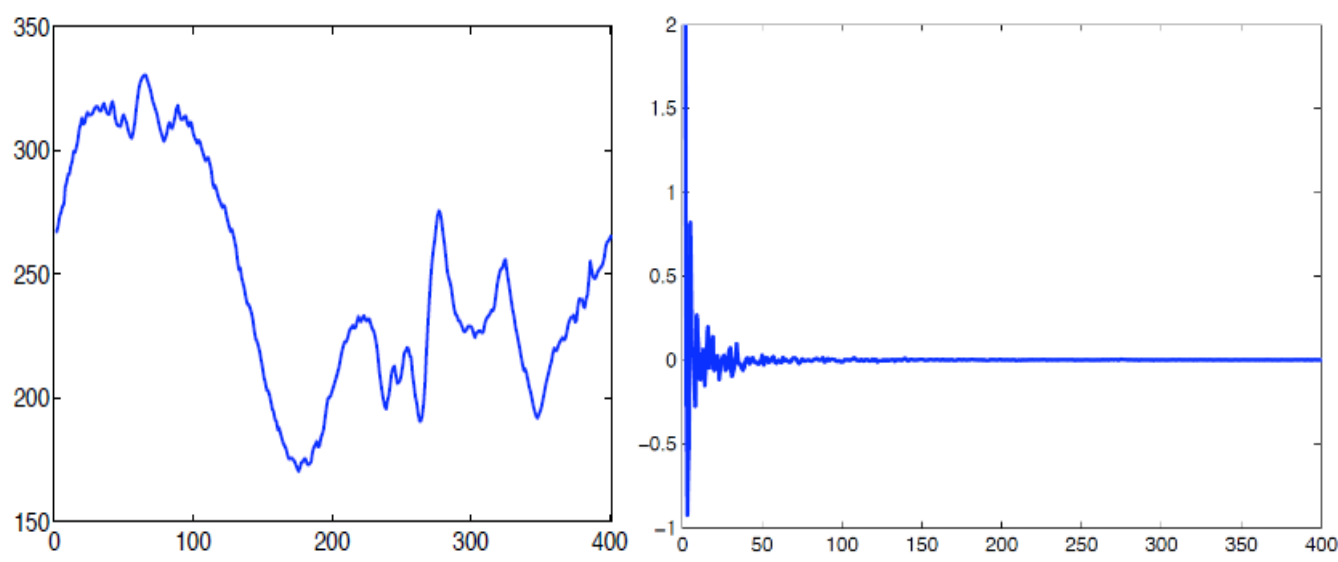
Figure 7

The recovery effect of a different sampling matrix

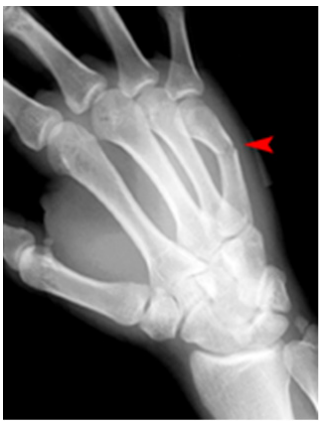

$\operatorname{Our}(d=0)$

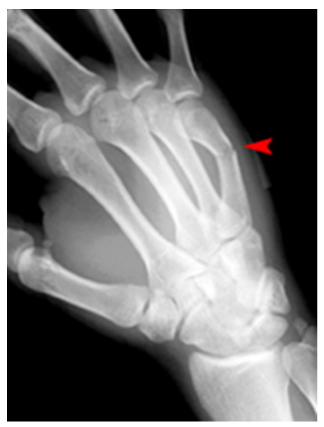

Z.M.Du $(d=0)$

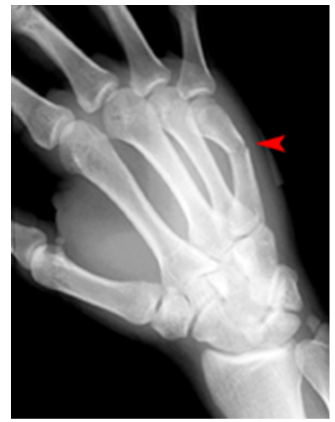

$\operatorname{pant}(d=0)$

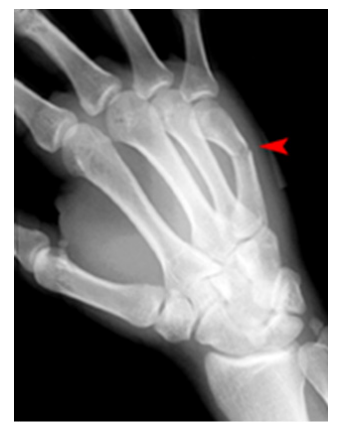

Donoho $(d=0)$

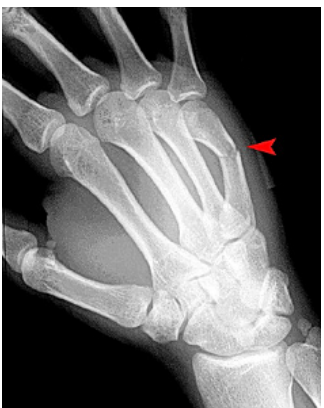

$\operatorname{Our}(d=0.8)$

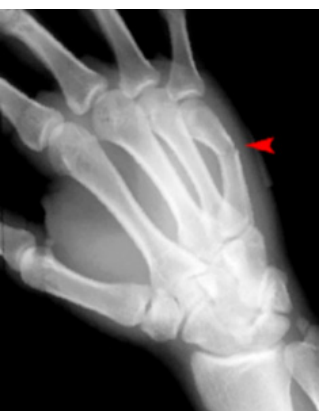

Z.M.Du $(d=2.0)$

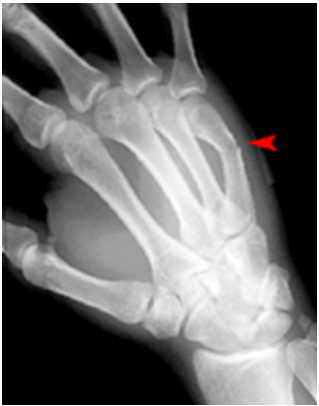

pant $(d=2.0)$

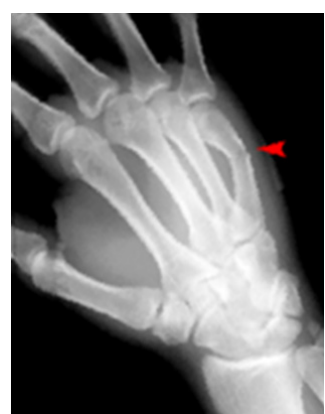

Donoho $(d=4.2)$ $\left(S_{76 \times 401}\right)$

$\left(S_{50 \times 401}\right)$

pressed sensing method. Our recovery method is for the compressed sensing method. We believe that compressed sensing and recovery methods could play an important role in the field of CT image processing.

\section{Conclusions}

In this paper, we recover the compressed CT images which are compressed by the compressed sensing method and achieve satisfactory results. However, this function is not convex in the whole space. The starting point is very important in the process of computing. So the future work will be emphasized to find out a convex function in the whole space. Thus, we can obtain the global optimal solution without considering the starting point.

\section{Acknowledgments}

This research is supported by the National key R\&D Program of China under Grant NO. 2018YFB0203901.

This work is also supported by National Natural Science Foundation of China (61731015, 61672103).

This work is supported by the National Natural Science Foundation of China (61402206), the Project Funded by China Postdoctoral Science Foundation (2016M601845, 2016M602941XB) and the Scientific Research Program Funded by Shaanxi Provincial Education Bureau (Program No. 16JK1497). We declare that there is no conflict of interests regarding the publication of this paper and would like to thank the anonymous reviewers for their valuable comments and suggestions. This work is also supported by The Key Research and Development Program of Shaanxi Province (No.2018ZDXM-GY-036). 


\section{References}

1. Beck, A., Teboulle, M. A Fast Iterative Shrinkage-Thresholding Algorithm for Linear Inverse Problems. SIAM Journal on Imaging Sciences, 2009, 2(1), 183-202. https:// doi.org/10.1137/080716542

2. Donoho, D. L. Compressed Sensing. IEEE Transactions on Information Theory, 2006, 52(4), 1289-1306. https:// doi.org/10.1109/TIT.2006.871582

3. Dou, H., Qi, Y., Wei, W., Song, H. A Two-Time-Scale Load Balancing Framework for Minimizing Electricity Bills of Internet Data Centers. Personal and Ubiquitous Computing, 2016, 20(5), 681-693. https://doi. org/10.1007/s00779-016-0941-9

4. Du., Z.-M., Ye, F.-Y., Shi, H., Zhu, G.-P. A Fast Recovery Method of 2D Geometric Compressed Sensing Signal. Circuits, Systems, and Signal Processing, 2015, 34(5), 1711-1724. https://doi.org/10.1007/s00034-014-9913-3

5. Ke, Q., Zhang, J., Song, H., Wan. C. Big Data Analytics Enabled by Feature Extraction Based on Partial Independence. Neurocomputing, 2017, 288, 3-10. https://doi. org/10.1016/j.neucom.2017.07.072

6. Lai, M. J., Xu, Y., Yin, W. Improved Iteratively Reweighted Least Squares for Unconstrained Smoothed \ell_q Minimization. SIAM Journal on Numerical Analysis, 2013,51(2), 927-957.https://doi.org/10.1137/110840364

7. Li, H.-A., Li, Z., Du, Z. A Reconstruction Method of Compressed Sensing 3D Medical Models Based on the Weighted 0-Norm. Computational and Mathematical Methods in Medicine, 2017, 7(2), 614-620. https://doi. org/10.1166/jmihi.2017.2030

8. Li, H.-A., Zhang, J., Kang, B., He, Y. A 3D Surface Reconstruction Algorithm Based on Medical Tomographic Images. Journal of Computational Information Systems, 2013, 19(9), 7873-7880.

9. Liu, S., Li, W., Du, D. Fractal Intelligent Privacy Protection in Online Social Network Using Attribute-Based Encryption Schemes. IEEE Transactions on Computational Social Systems, 2018, 5(3), 736-747. https://doi. org/10.1109/TCSS.2018.2855047

10. Mohimani, H., Babaie-Zadeh, M., Jutten, C. A Fast Approach for Overcomplete Sparse Decomposition Based on Smoothed Norm. IEEE Transactions on Signal Processing, 2009, 57(1), 289-301. https://doi.org/10.1109/ TSP.2008.2007606

11. Pant, J. K., Lu, W. S., Antoniou, A. New Improved Algorithms for Compressive Sensing Based on Norm.
IEEE Transactions on Circuits and Systems II: Express Briefs, 2014, 61(3), 198-202. https://doi.org/10.1109/ TCSII.2013.2296133

12. Pant, J. K., Lu, W. S., Antoniou, A. Reconstruction of Sparse Signals by Minimizing a Re-weighted Approximate 10 -Norm in the Null Space of the Measurement Matrix. 53rd IEEE International Midwest Symposium on Circuits and Systems (MWSCAS), 2010, 430-433. https://doi.org/10.1109/MWSCAS.2010.5548758

13. Pant, J. K., Lu, W. S., Antoniou, A. Recovery of Sparse Signals from Noisy Measurements Using an $1 \mathrm{p}$ Regularized Least-Squares Algorithm. 2011 IEEE Pacific Rim Conference on Communications, Computers and Signal Processing (PacRim), 2011, 48-53. https://doi. org/10.1109/PACRIM.2011.6032866

14. Pant, J. K., Lu, W. S., Antoniou, A. Unconstrained Regularized $1 \mathrm{p}$-Norm Based Algorithm for the Reconstruction of Sparse Signals. 2011 IEEE International Symposium on Circuits and Systems (ISCAS), 2011, 1740-1743. https://doi.org/10.1109/ISCAS.2011.5937919

15. Qiao, Y.-N., Yong, Q., Di, H. Tensor Field Model for Higher-Order Information Retrieval. Tensor Field Model for Higher-Order Information Retrieval, 2011, 84, 23032313. https://doi.org/10.1016/j.jss.2011.06.057

16. Srivastava, H. M., Zhang, Y., Wang, L., Shen, P., Zhang, J. A Local Fractional Integral Inequality on Fractal Space Analogous to Anderson's Inequality: Abstract and Applied Analysis. Hindawi Publishing Corporation, 2014, 46(8), 5218-5229.

17. Wang, P., Qi, Y., Liu, X. Power-Aware Optimization for Heterogeneous Multi-tier Clusters. Journal of Parallel and Distributed Computing, 2014, 74(1), 2005-2015. https://doi.org/10.1016/j.jpdc.2013.09.003

18. Wang, X., Qi, Y., Wang, Z., Chen, Y., Zou, Y. Design and Implementation of SecPod: A Framework for Virtualization-Based Security Systems. 2019, 16(1), 44-57. https://doi.org/10.1109/TDSC.2017.2675991

19. Wei, W., Fan, X., Song, H., Fan, X., Yang, J. Imperfect Information Dynamic Stackelberg Game Based Resource Allocation Using Hidden Markov for Cloud Computing. IEEE Transactions on Services Computing, 2016, 11(1), 78-89. https://doi.org/10.1109/TSC.2016.2528246

20. Wei, W., Fan, X., Song, H., Wang, H. Video Tamper Detection Based on Multi-Scale Mutual Information. Multimedia Tools \& Applications, 2017, 1-18. https:// doi.org/10.1007/s11042-017-5083-1 
21. Wei, W., Qiang, Y., Zhang, J. A Bijection Between Lattice-Valued Filters and Lattice-Valued Congruences in Residuated Lattices. Mathematical Problems in Engineering, 2013, 36(8), 4218-4229. https://doi. org/10.1155/2013/908623

22. Wei, W., Song, H., Li, W., Shen, P., Vasilakos, A. Gradient-Driven Parking Navigation Using a Continuous Information Potential Field Based on Wireless Sensor Network. Information Sciences, 2017, 408(C), 100-114. https://doi.org/10.1016/j.ins.2017.04.042

23. Wei, W., Song, H., Wang, H., Fan, X. Research and Simulation of Queue Management Algorithms in Ad Hoc Networks Under DDoS Attack. IEEE Access, 2017, 5, 27810-2781\%. https://doi.org/10.1109/ACCESS.2017.2681684

24. Wei, W., Sun, Z., Song, H., Wang, H., Fan, X., Chen, X. Energy Balance-Based Steerable Arguments Coverage Method in WSNs. IEEE Access, 2017, 6(99), 3376633773. https://doi.org/10.1109/ACCESS.2017.2682845

25. Wei, W., Xu, Q., Wang, L., Hei, X. H., Shen, P., Shi, W., Shan, L. GI/Geom/1 Queue Based on Communication Model for Mesh Networks. International Journal of Communication Systems, 2014, 27(11), 3013-3029. https://doi.org/10.1002/dac.2522

26. Wei, W., Yang, X. L., Shen, P. Y., Zhou, B. Holes Detection in Anisotropic Sensornets: Topological Methods. International Journal of Distributed Sensor Networks, 2012, 8(10). https://doi.org/10.1155/2012/135054

27. Wei, W., Yang, X. L., Zhou, B., Feng, J., Shen, P. Y. Combined Energy Minimization for Image Reconstruction from Few Views. Mathematical Problems in Engineering, 2012.https://doi.org/10.1155/2012/154630
28. Xi, M., Qi, Y., Wu, K., Zhao, J., Li, M. Using Potential to Guide Mobile Nodes in Wireless Sensor Networks. Ad Hoc \& Sensor Wireless Networks, 2011, 12(3-4), 229251.

29. Yan, J., Qi, Y., Rao, Q. Detecting Malware with an Ensemble Method Based on Deep Neural Network. Security and Communication Networks, 2018. https://doi. org/10.1155/2018/7247095

30. Yang, L., Qi, Y., Han, J., Wang, C., Liu, Y. Shelving Interference and Joint Identification in Large-Scale RFID Systems. IEEE Transactions on Parallel and Distributed Systems, 2015, 26(11), 3149-3159. https://doi. org/10.1109/TPDS.2013.276

31. Yong, Q. Information Potential Fields Navigation in Wireless Ad-Hoc Sensor Networks. Sensors, 2011, 11(5), 4794-4807. https://doi.org/10.3390/s110504794

32. Zhang, J., Wei, W., Damasevicius, R., Wozniak, M. Adaptive Independent Subspace Analysis of Brain Magnetic Resonance Imaging Data. IEEE Access, 2019, 7(1), 12252-12261. https://doi.org/10.1109/ACCESS.2019.2893496

33. Zhang, J., Wei, W., Połap, D., Woźniak, M., Kośmider, L., Damaševicius, R. A Neuro-Heuristic Approach for Recognition of Lung Diseases from X-Ray Images. Author Links Open Overlay Panel, Expert Systems with Applications, 2019, 126, 218-232. https://doi.org/10.1016/j. eswa.2019.01.060

34. Zheng, P., Qi, Y., Zhou, Y., Chen, P., Zhan, J., Lyu, M. R.T. An Automatic Framework for Detecting and Characterizing the Performance Degradation of Software Systems. IEEE Transactions on Reliability, 63(4), 927-943. https://doi.org/10.1109/TR.2014.2338255 